\title{
RELATIONS, HOMOGENEITY AND GROUP QUOTIENTS
}

\author{
M. W. WARNER \\ (Received 25 February 1980) \\ Communicated by W. D. Wallis
}

\begin{abstract}
A set with a relation is isomorphic to a group quotient under the condition described as weak homogeneity, and to the quotient of a group with relation preserved by right and left translations if the homogeneity is strengthened. A method of constructing these group quotients and, furthermore, all such very homogeneous spaces, is described and an illustrative example given.
\end{abstract}

1980 Mathematics subject classification (Amer. Math. Soc.): primary 05 C 25, 05 C 20; secondary $68 \mathrm{D} 30$.

\section{Introduction}

1.1. Given a set $Q$ with a relation $\nu \subseteq Q \times Q$, we define a relation $\nu$ on the function set $Q^{Q}$ and in particular on the group $G^{*}$ of set bijections of $Q$ to itself (see the coarse function space tolerance (Muir and Warner (1979), (1980))). The relation $\nu$ on $G^{*}$ is preserved by right translations. Under the condition on $Q$ described as weak homogeneous, namely for all $q \nu q^{\prime}$, there exists $g \nu$ identity such that $g\left(q^{\prime}\right)=q, g \in G^{*}$, we establish an isomorphism (a $\nu$-preserving bijection whose inverse also preserves $\nu$ ) between $Q$ and a quotient of $G^{*}$ by a (not necessarily normal) subgroup.

Conversely, every such right translation group $\left(G^{*}, \nu\right)$ and, furthermore, every group quotient $G^{*} / H^{*}$ with the coinduced relation from $\nu$ is weak homogeneous.

1.2. However (Section 3), when $Q$ is also homogeneous, $G^{*}$ can be replaced by the group $G$ of isomorphisms of $Q$ to itself. Then $G$ is a $\nu$-group, that is $\nu$ is

OCopyright Australian Mathematical Society 1981 
preserved by both right and left translations. In the isomorphism theorem described in 1.1 the condition weak homogeneous must be replaced by very homogeneous, namely the bijection $g$ in 1.1 must be an isomorphism (see Muir and Warner (1980)). Then every $\nu$-group $(G, \nu)$ and every group quotient $G / H$ with the coinduced relation is homogeneous and very homogeneous. If $H$ is normal then $(G / H, \nu)$ is also a $\nu$-group.

1.3. Finally we observe that in a $\nu$-group $G$ the subsets $M_{\nu}=\{g \in G ; 1 \nu g\}$, $M_{\nu}^{\prime}=\{g \in G ; g \nu 1\}$ are self-conjugate. This enables us to construct $\nu$-groups by stipulating $M_{\nu}$ and using homogeneity. All homogeneous and very homogeneous spaces are then obtained as group quotients. In Section 4 we thus construct a $\nu$-group and examine some of its quotients.

1.4. Some of the foregoing generalizes results by Muir and Warner (1980) on sets with a reflexive symmetric relation called a tolerance. The notion of relation has itself been generalized recently by Muir in the context of neural nets where the relations may be of different strengths and either excitatory or inhibitory.

1.5. We emphasize the bijective group $G^{*}$ rather than restricting ourselves to the isomorphism group $G$ since this is precisely what is required in the theory of finite automata (discussed by the author in a forthcoming paper). Given an input set $X$ acting by right translation on the state space $Q$, define the relation $\nu$ on $Q$ by $q_{1} \nu q_{2}$ if and only if there exists $x \in X$ such that $q_{2}=q_{1} \cdot x$, that is $q_{1}$ is related to $q_{2}$ if and only if there exists an elementary input of $X$ which sends $q_{1}$ to $q_{2}$. This is an improvement on Arbib's (Arbib (1967)) tolerance on $Q$. The inputs are not in general morphisms of $(Q, v)$, and the group generated by the permutation inputs is a right translation group.

1.6. We do not, however, restrict ourselves to finite sets $Q$. Thus all our conclusions are valid for $Q$ (and therefore $G^{*}, G$ ) infinite. When $Q$ is finite, $(Q, \nu)$ can be thought of as a directed graph. Such a graph is drawn for the example of Section 4.

Except when there is any possibility of confusion all relations will be written $\nu$, even in the function space $Q^{Q}$.

\section{Weak homogeneity}

2.1. Let $(Q, \nu)$ be a set with relation $\nu \subseteq Q \times Q, P$ any set.

Definition. The relation $\nu$ in the function space $Q^{P}$ is defined by $f \nu f^{\prime} \Leftrightarrow$ $f(p) \nu f^{\prime}(p)$ for all $p \in P$. 
2.2 Definition. The function $f:(Q, v) \rightarrow\left(Q^{\prime}, \nu^{\prime}\right)$ is a morphism if and only if whenever $q_{1} \nu q_{2}$ then $f\left(q_{1}\right) \nu f\left(q_{2}\right)$. A bijection which is a morphism and whose inverse is a morphism is an isomorphism.

2.3. Let the group $G^{*}$ of bijections of $Q$ to itself be given the relation $\nu$ induced from $Q^{Q}$.

LEMMA. $\nu$ is preserved by right translations in $G^{*}$.

Proof. $g_{1} \nu g_{2}\left(g_{1}, g_{2} \in G^{*}\right) \Rightarrow g_{1}(q) \nu g_{2}(q)$ for all $q \in Q \Rightarrow$ $g_{1} \bar{g}\left(q^{\prime}\right) \nu g_{2} \bar{g}\left(q^{\prime}\right)$ for all $\bar{g} \in G^{*}, q^{\prime} \in Q$. Thus $g_{1} \bar{g} \nu g_{2} \bar{g}$.

Such a group $\left(G^{*}, \nu\right)$ is called a right translation group.

2.4. For a fixed $q_{0} \in Q$, let $H^{*}$ be the subgroup of $G^{*}$ which fixed $q_{0}$, and define an equivalence relation $\sim$ on $G^{*}$ by $g_{1} \sim g_{2}$ if and only if $g_{1}\left(q_{0}\right)=g_{2}\left(q_{0}\right)$. Then the quotient $G^{*} / \sim$ may be thought of as the set $G^{*} / H^{*}$ of left cosets of $H^{*}$, and is not necessarily a group. If [ ] denotes equivalence class with respect to $\sim$, the coinduced relation $\nu$ on $G^{*} / H^{*}$ is defined by $\left[g_{1}\right] \nu\left[g_{2}\right]$ if and only if there exist $g_{1}^{\prime} \sim g_{1}, g_{2}^{\prime} \sim g_{2}$ such that $g_{1}^{\prime} \nu g_{2}^{\prime}$. In terms of cosets, $g_{1} H^{*} \nu g_{2} H^{*}$ if and only if there exist $h_{1}, h_{2} \in H^{*}$ such that $g_{1} h_{1} \nu g_{2} h_{2}$, that is if there exists $h \in H^{*}$ such that $g_{1} h v g_{2}$ (Lemma 2.3).

2.5 Definition. $(Q, v)$ is weak homogeneous if whenever $q \nu q^{\prime}$, there exists $g \nu 1$ such that $g\left(q^{\prime}\right)=q$ where $q, q^{\prime} \in Q, g \in G^{*}$, and 1 is the identity in $G^{*}$.

2.6. Define $\tilde{\varphi}: G^{*} / H^{*} \rightarrow Q$ by $\tilde{\varphi}[g]=g\left(q_{0}\right)$. Then $\tilde{\varphi}$ is well defined since $[g]=\left[g^{\prime}\right] \Rightarrow g\left(q_{0}\right)=g^{\prime}\left(q_{0}\right)$.

THEOREM. $\tilde{\varphi}$ is a bijective morphism. If $(Q, \nu)$ is weak homogeneous $\tilde{\varphi}$ is an isomorphism.

Proof, (i) $\tilde{\varphi}$ is bijective $\left(\tilde{\varphi}[g]=\tilde{\varphi}\left[g^{\prime}\right] \Rightarrow g\left(q_{0}\right)=g^{\prime}\left(q_{0}\right) \Rightarrow[g]=\left[g^{\prime}\right]\right)$, and $\forall q \in Q, \exists g \in G^{*}$ such that $g\left(q_{0}\right)=q$.

(ii) $\left[g_{1}\right] \nu\left[g_{2}\right] \Rightarrow \exists g_{1}^{\prime} \sim g_{1}, g_{2}^{\prime} \sim g_{2}$ such that

$$
g_{1}^{\prime} \vee g_{2}^{\prime} \Rightarrow g_{1}^{\prime}\left(q_{0}\right) \vee g_{2}^{\prime}\left(q_{0}\right) \Rightarrow g_{1}\left(q_{0}\right) \vee g_{2}\left(q_{0}\right)
$$

that is $\tilde{\varphi}\left[g_{1}\right] \nu \tilde{\varphi}\left[g_{2}\right]$.

(iii) Now let $Q$ be weak homogeneous, and let $q \nu q^{\prime}, q, q^{\prime} \in Q$. Then there exist $g, g^{\prime} \in G^{*}$ such that $g\left(q_{0}\right)=q, g^{\prime}\left(q_{0}\right)=q^{\prime}$, so $g\left(q_{0}\right) \vee g^{\prime}\left(q_{0}\right)$. Hence there 
exists $h \nu 1$ such that $h g^{\prime}\left(q_{0}\right)=g\left(q_{0}\right)$. Thus $\left[h g^{\prime}\right]=[g]$ and $\left[h g^{\prime}\right] \nu\left[g^{\prime}\right]$ since $h g^{\prime} \nu g^{\prime}$ by right translation. This means that $\tilde{\varphi}^{-1}(q) \nu \tilde{\varphi}^{-1}\left(q^{\prime}\right)$.

2.7 LEMMA. Every right translation group $\left(G^{*}, \nu\right)$ and subgroup $H$, yield a weak homogeneous set $\left(G^{*} / H^{*}, \nu\right)$ where $\nu$ is coinduced on the left cosets of $G^{*} / H^{*}$ as in Section 2.4 .

ProOF. Every $\bar{g} \in G^{*}$ gives rise to a well-defined function $\bar{g}: G^{*} / H^{*} \rightarrow G^{*} / H^{*}, \bar{g}\left(g H^{*}\right)=\bar{g} g H^{*}, \quad$ for $g_{1} H^{*}=g_{2} H^{*} \Rightarrow \bar{g} g_{1} H^{*}=\bar{g} g_{2} H^{*}$. Also $\bar{g}$ is bijective $\left(\bar{g}\left(g_{1} H^{*}\right)=\bar{g}\left(g_{2} H^{*}\right) \Rightarrow g_{1} H^{*}=g_{2} H^{*}\right.$; and $g H^{*}$ is the image under $\bar{g}$ of $\left.\bar{g}^{-1} g H^{*}\right)$.

Let $g_{1} H^{*} \nu g_{2} H^{*}$, that is $\exists h \in H^{*}$ such that $g_{1} h \nu g_{2}$. Then $g_{1} h g_{2}^{-1} \nu 1$ (right translation), so for all $g H^{*}, g_{1} h g_{2}^{-1}\left(g H^{*}\right) \nu g H^{*}$, while $g_{1} h g_{2}^{-1}\left(g_{2} H^{*}\right)=g_{1} h H^{*}=$ $g_{1} H^{*}$. So $g_{1} h g_{2}^{-1}$ is the required bijectory $\bar{g} \nu 1$ of $G^{*} / H^{*}$ which is therefore weak homogeneous.

2.8 COROLlARY 1. Every right translation group $\left(G^{*}, \nu\right)$ is weak homogeneous.

COROLlaRy 2. In Theorem $2.6 \tilde{\varphi}$ is an isomorphism if and only if $(Q, \nu)$ is weak homogeneous.

\section{Homogeneity}

3.1 Definition. $(Q, \nu)$ is homogeneous if for all $q, q^{\prime} \in Q$, there exists an isomorphism $g$ of $Q$ such that $g(q)=q^{\prime}$.

Definition. $(Q, v)$ is very homogeneous if whenever $q \nu q^{\prime}$, there exists an isomorphism $g \nu 1$ such that $g\left(q^{\prime}\right)=q$.

LemMa. Very homogeneous implies homogeneous when $Q$ is connected, that is for all $q, q^{\prime} \in Q$ there exist $q_{0}, q_{1}, \ldots, q_{n} \in Q$ such that $q=q_{0}, q^{\prime}=q_{n}$ and $q_{i} v q_{i+1}, i=0, \ldots, n-1$.

3.2 Definition. $(G, \nu)$ is a $\nu$-group if it is both a left and right translation group, that is $g_{1} \nu g_{2} \Rightarrow \bar{g} g_{1} \nu \bar{g} g_{2}$ and $g_{1} \bar{g} \nu g_{2} \bar{g}$ for all $\bar{g} \in G$.

Note that it follows that $g_{1} \nu g_{2} \Rightarrow g_{2}^{-1} \nu g_{1}^{-1}$.

LeMmA. The isomorphism group $G$ of a set $(Q, v)$ is a $\nu$-group. 
ProOF.

$$
\begin{aligned}
g_{1} \vee g_{2} & \Rightarrow g_{1}(q) \vee g_{2}(q) \quad \text { for all } q \in Q \\
& \Rightarrow \bar{g} g_{1}(q) \vee \bar{g} g_{2}(q)
\end{aligned}
$$

since every $\bar{g} \in G$ is a morphism, so $\bar{g} g_{1} \nu \bar{g} \bar{g}_{2}$. Application of Lemma 2.3 completes the proof.

3.3 LEMMA. The group quotient $(G / H, \nu)$ of a $\nu$-group $G$ by a subgroup $H$ is very homogeneous.

Proof. This follows exactly that of Lemma 2.7, but it is necessary to prove that $\bar{g}: G / H \rightarrow G / H$ is an isomorphism for all $\bar{g} \in G$. Now

$$
\begin{aligned}
g_{1} H \nu g_{2} H & \Leftrightarrow g_{1} h \nu g_{2} \quad \text { for some } h \in H \\
& \Leftrightarrow \bar{g} g_{1} h \nu \bar{g} g_{2} \quad \text { (left translation) } \\
& \Leftrightarrow \bar{g}\left(g_{1} H\right) \nu \bar{g}\left(g_{2} H\right) .
\end{aligned}
$$

COROLlARY. Every $\nu$-group is very homogeneous.

3.4. Every $\nu$-group $(G, \nu)$ is homogeneous since for all $g_{1}, g_{2} \in G, g_{2} g_{1}^{-1}$ : $G \rightarrow G$ is the required isomorphism. Similarly $(G / H, \nu)$ is homogeneous.

3.5. Let $(G, \nu)$ be the isomorphism group of $(Q, \nu)$, and let $H$ be the subgroup of $G$ which fixes $q_{0}$.

THEOREM. If and only if $(Q, \nu)$ is homogeneous and very homogeneous, then $\tilde{\varphi}$ : $G / H \rightarrow Q$, defined $\tilde{\varphi}[g]=g\left(q_{0}\right)$ is an isomorphism.

Proof. Exactly as in Theorem 2.6, concluding with an application of 3.3 and 3.4 .

3.6 LEMMA. If $H$ is a normal subgroup of the $\nu$-group $(G, \nu)$, then $(G / H, \nu)$ is a $\nu$-group.

Proof. The group operation is given by $(g H)(k H)=g k H, g, k \in G$. Then

$$
\begin{aligned}
g_{1} H \nu g_{2} H & \Rightarrow \bar{g} g_{1} H \nu \bar{g} g_{2} H \quad \text { (Lemma 3.3) } \\
& \Rightarrow(\bar{g} H)\left(g_{1} H\right) \nu(\bar{g} H)\left(g_{2} H\right)
\end{aligned}
$$


And

$$
\begin{aligned}
g_{1} H \nu g_{2} H & \Rightarrow \exists h \in H \quad \text { such that } g_{1} h \nu g_{2} \\
& \Rightarrow g_{1} h \bar{g} \nu h_{2} \bar{g} \\
& \Rightarrow g_{1} \bar{g} h^{\prime} \nu g_{2} \bar{g} \quad \text { for some } h^{\prime} \in H \\
& \Rightarrow g_{1} \bar{g} H \nu g_{2} \bar{g} H \\
& \Rightarrow\left(g_{1} H\right)(\bar{g} H) \nu\left(g_{2} H\right)(\bar{g} H)
\end{aligned}
$$

for all $g \in G$.

3.7 Lemma. If and only if $(G, \nu)$ is a $\nu$-group, the subsets $M_{\nu}=\{g \in G ; 1 \nu g\}$, $M_{\nu}^{\prime}=\{g \in G ; g \nu 1\}$ are self-conjugate.

ProOF.

$$
\begin{aligned}
& 1 \nu g \Leftrightarrow 1=\bar{g} \bar{g}^{-1} \nu \bar{g} g \bar{g}^{-1}, \\
& g \nu 1 \Leftrightarrow \bar{g} g \bar{g}^{-1} \nu \bar{g} \bar{g}^{-1}=1
\end{aligned}
$$

for all $\bar{g} \in G$.

\section{Example}

4.1. The conclusion of Lemma 3.7 enables us to construct a $\nu$-group $G$ by specifying a self-conjugate subset $M_{v}$, to which the identity is related. Any group $G$ thus equipped receives the rest of its structure by homogeneity, the 'neighbourhood' of $g \in G$ being $g\left(M_{v}\right)$.

By Theorem 3.5 every very homogeneous connected space can then be obtained as a quotient of such a $\boldsymbol{\nu}$-group by a subgroup.

4.2. The following example places a $\nu$-structure on $A_{4}$, the alternating group on four elements. We have chosen a non-abelian group so that self-conjugacy is not trivial. $A_{4}$ is also large enough to provide both a normal subgroup and self-conjugate subsets which are not groups.

4.3. Table 1 is the group table of $A_{4}$, while Diagram 1 is the directed graph of $\left(A_{4}, \nu\right)$ generated by selecting $M_{v}=\{B, E, H, L\}$. The remaining relations are then found by translation.

4.4. The normal subgroup $K_{4}=\{I, C, F, J\}$ yields a factor group of order 3 with the cyclic coinduced relation illustrated graphically in Diagram 2, while the non-normal subgroup $\{I, F\}=Z$ has a quotient set whose graph is illustrated in Diagram 3. It is obvious that both graphs are very homogeneous. 
4.5. We note the duality which exists between $M_{\nu}$ and $M_{\nu}^{\prime}=\{A, D, G, K\}$ which is also self-conjugate. Observe also that if $K_{4}$ were taken as $M_{v^{*}}$, defining a relation $\nu^{*}$ on $G$ then $A_{4} / K_{4}$ with the co-induced relation from $\nu^{*}$ is the totally disconnected group $\left\{K_{4}, A K_{4}, B K_{4}\right\}$ in which every element is related to itself only.

\section{TABLE 1}

Group table for $A_{4}$

\begin{tabular}{c|ccccccccccc}
$I$ & $A$ & $B$ & $C$ & $D$ & $E$ & $F$ & $G$ & $H$ & $J$ & $K$ & $L$ \\
\hline$A$ & $B$ & $I$ & $D$ & $E$ & $C$ & $G$ & $H$ & $F$ & $K$ & $L$ & $J$ \\
$B$ & $I$ & $A$ & $E$ & $C$ & $D$ & $H$ & $F$ & $G$ & $L$ & $J$ & $K$ \\
$C$ & $G$ & $L$ & $I$ & $K$ & $H$ & $J$ & $A$ & $E$ & $F$ & $D$ & $B$ \\
$D$ & $H$ & $J$ & $A$ & $L$ & $F$ & $K$ & $B$ & $C$ & $G$ & $E$ & $I$ \\
$E$ & $F$ & $K$ & $B$ & $J$ & $G$ & $L$ & $I$ & $D$ & $H$ & $C$ & $A$ \\
$F$ & $K$ & $E$ & $J$ & $G$ & $B$ & $I$ & $D$ & $L$ & $C$ & $A$ & $H$ \\
$G$ & $L$ & $C$ & $K$ & $H$ & $I$ & $A$ & $E$ & $J$ & $D$ & $B$ & $F$ \\
$H$ & $J$ & $D$ & $L$ & $F$ & $A$ & $B$ & $C$ & $K$ & $E$ & $I$ & $G$ \\
$J$ & $D$ & $H$ & $F$ & $A$ & $L$ & $C$ & $K$ & $B$ & $I$ & $G$ & $E$ \\
$K$ & $E$ & $F$ & $G$ & $B$ & $J$ & $D$ & $L$ & $I$ & $A$ & $H$ & $C$ \\
$L$ & $C$ & $G$ & $H$ & $I$ & $K$ & $E$ & $J$ & $A$ & $B$ & $F$ & $D$
\end{tabular}

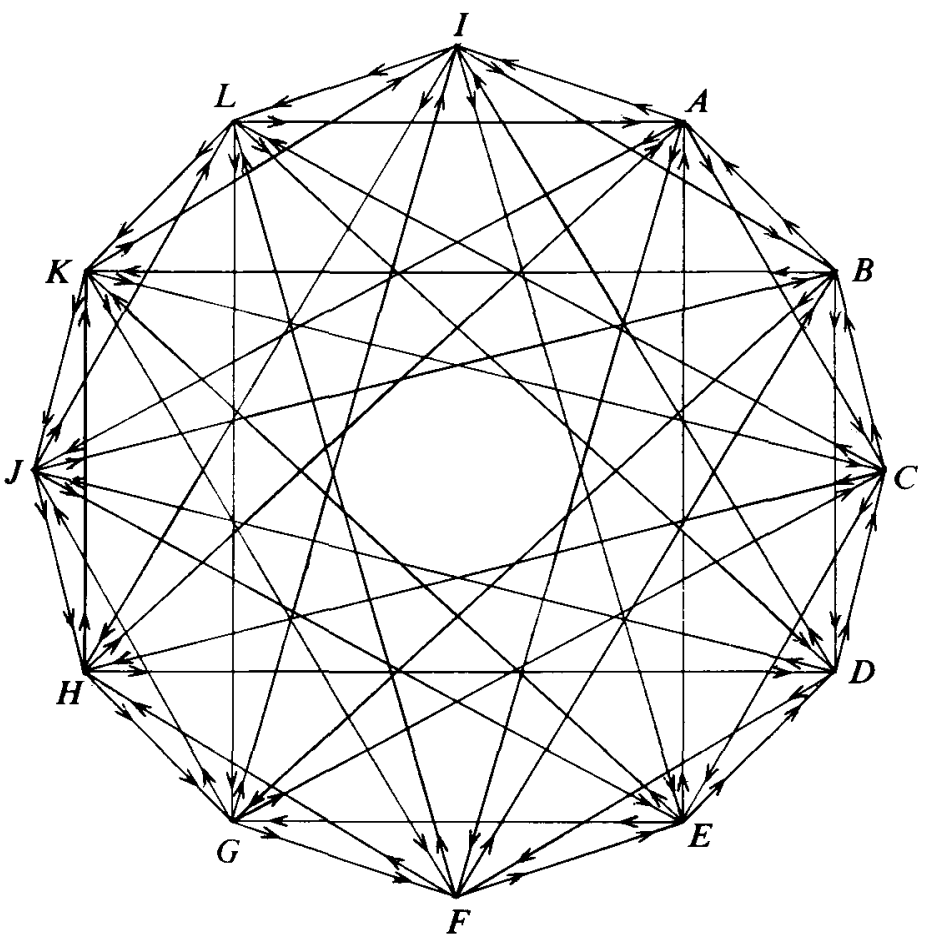

Diagram 1. The graph of $\left(A_{4}, v\right)$ 


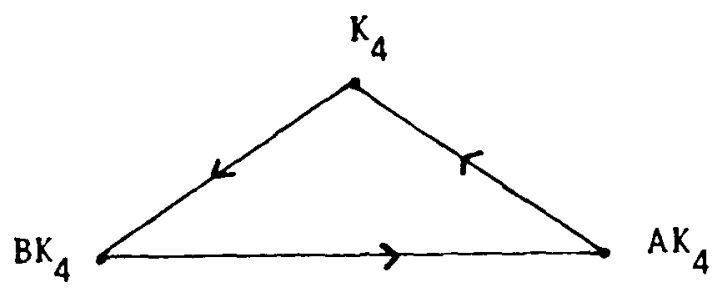

Diagram 2. $\left(A_{4} / K_{4}, \nu\right)$

$\left(A K_{4}\right)\left(B K_{4}\right)=(A B) K_{4}=K_{4}=\{I, C, F, J\} . A K_{4} \vee K_{4}$ since $A v 1$, etc. The cosets $A K_{4} B K_{4}$ are the self-conjugate subsets $M_{\eta}, M_{;}^{\prime}$ respectively.

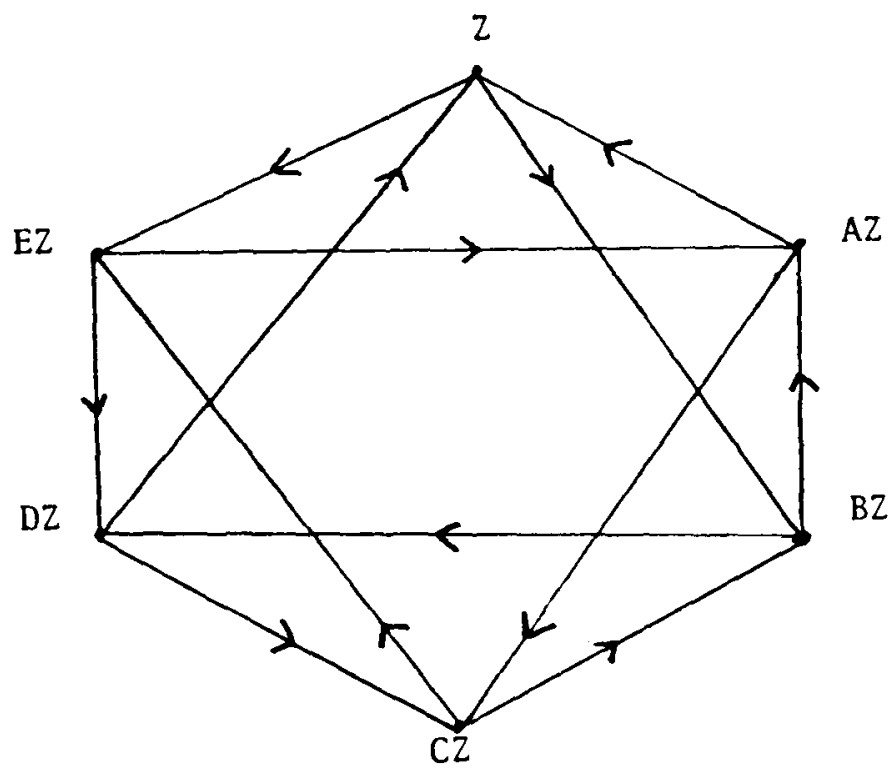

Diagram 3. $\left(A_{4} / Z, \nu\right)$

$$
\begin{array}{rlrl}
Z & =\{I, F\}, & A Z=\{A, G\}, & B Z=\{B, H\} \\
C Z=\{C, J\}, & D Z=\{D, K\}, & E Z=\{E, L\}
\end{array}
$$

I am grateful to A. Muir for interesting and helpful discussions on the subject matter of this paper.

\section{References}

M. A. Arbib, 'Tolerance automata', Kybernetika (Prague) 3, 223-233.

A. Muir and M. W. Warner (1979), 'Tolerability, tolerance and automata', Research Memorandum 12, Department of Mathematics, The City University, London. 
A. Muir and M. W. Warner (1980), 'Homogeneous tolerance spaces', Czechoslovak Math. J. 30 (105), 47-55.

Department of Mathematics

The City University

Northampton Square

London ECl

United Kingdom 